\title{
De l'incidentalome à la découverte secondaire
}

$>$ Le terme incidentalome, créé en 1982, s'est, depuis, diffusé dans la littérature médicale. II ne Cédric Gesbert ${ }^{1}$, Cécile Torregrosa ${ }^{1}$, Marie-France Mamzer ${ }^{1,2}$ semble cependant pas exister de définition précise pour décrire ce qu'est un incidentalome. Dans les usages initiaux, l'incidentalome désignait une masse détectée à l'occasion d'un examen d'imagerie prescrit à visée diagnostique sans qu'un lien ne soit déterminé avec le motif de réalisation de l'examen. La qualification d' « incidentalome » de cette masse n'apportait aucune précision sur sa nature, celleci pouvant être située dans de nombreuses zones anatomiques, être sécrétante ou non, être bénigne ou maligne... Aujourd'hui, le terme d'incidentalome porte une dimension beaucoup plus large, semblant recouvrir la notion de découverte fortuite, qu'elle soit radiologique, biologique ou génétique. Cet usage évolutif du terme démontre son caractère heuristique. II est le signe d'une modification de la médecine moderne qui hésite entre une clinique des patients et une clinique des données. Les découvertes fortuites sont un phénomène connu et anticipé par les radiologues. Ces découvertes ne sont donc plus fortuites, ni même inattendues, mais bien secondaires à l'usage de la technologie en santé. <

Le terme incidentalome résume, en lui-même, une problématique médicale née de l'usage du scanner à visée diagnostique dans les années 1980, qui, laissant apparaître des tissus et organes jusqu'alors « transparents » aux rayons $X$, en proposaient des images inédites, en coupe et in situ, à l'origine d'une «nouvelle anatomie » radiologique.

Certains organes, dont les glandes surrénales, jusqu'alors invisibles de l'extérieur, révélaient régulièrement au regard des radiologues, des masses cliniquement asymptomatiques, qui posaient donc aux médecins le problème de leur signification clinique. La principale préoccupation des médecins était alors l'indication chirurgicale de ces masses $[1,2]$. C'est dans ce contexte de controverse sur la conduite

Vignette (Photo @ Marie-France Mamzer).

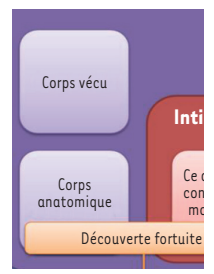

à tenir devant ces

images, qu'un chirurgien et un radiologue ont créé, en 1982, le néologisme « incidentaloma » pour désigner ces images de signification inconnue, de découverte inattendue, et de localisation surrénalienne, baptisées à cette occasion «adrenal incidentalomas » [3]. L'usage de ce terme en tant que «mot-clé » d'indexation des publications médicales et scientifiques resta confidentiel pendant une dizaine d'années avant son essor à l'orée des années 2000 (Figure 1), témoignant de son intégration dans le vocabulaire médical de trois disciplines : la radiologie, la chirurgie et l'endocrinologie.

Malgré de vives critiques sur l'utilisation expansive d'un nouveau terme technique pour lequel il n'existait pas de définition consensuelle, rendant pour certains son usage inutile, regrettable, voire à bannir [4], ce néologisme s'est pourtant imposé avant de devenir viral dans la littérature médico-scientifique. Dans un premier temps, toutes les masses de signification clinique incertaine, découvertes fortuitement à l'intérieur d'un organe silencieux par un examen d'imagerie, ont pu être désignées par ce terme, quel que soit leur siège anatomique. L'usage du terme s'est, dans un second temps, étendu au domaine de la génétique, suggérant la fonction heuristique ${ }^{1}$ de ce néologisme médical...

\section{D’une problématique à un néologisme}

Créé dans le cadre du débat scientifique engagé autour de la conduite à tenir lors de la découverte par le scanner de masses surrénaliennes de nature indétermi-

\footnotetext{
Qui sert à la découverte.
} 


\section{Évolution des parutions comprenant le terme « incidentaloma » sur PubMed}

120

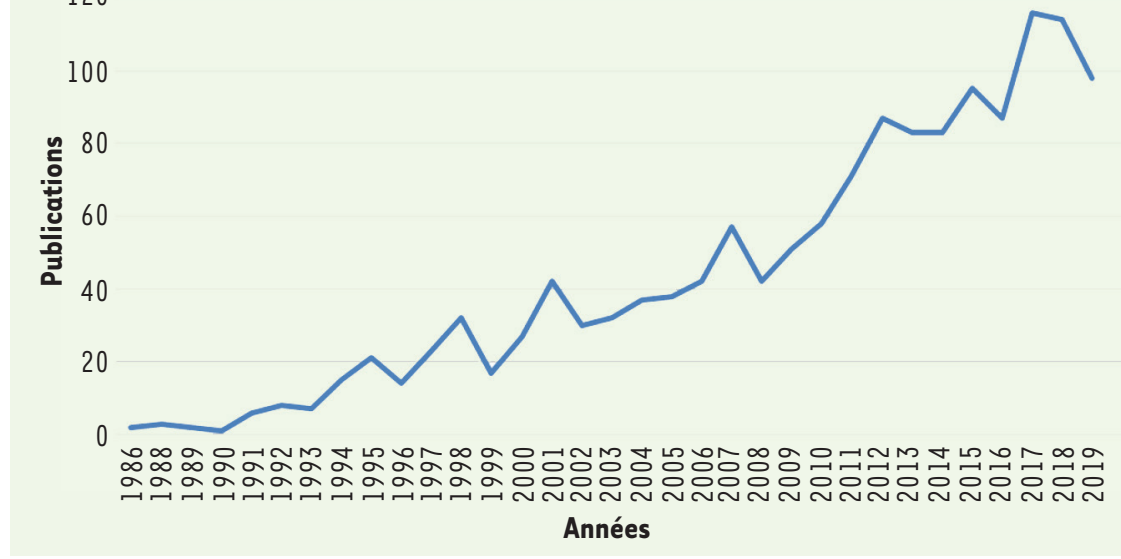

Figure 1. Chronologie des publications répondant au mot-clé « incidentaloma » sur PubMed.

une définition des incidentalomes : " an incidentally discovered mass or lesion, detected by $C T, M R I$, or other imaging modality performed for an unrelated reason $»^{2}[18]$. Cette définition, consensuelle pour ce groupe d'experts, entérinait donc l'existence (et donc l'utilité) d'un terme technique spécifique pour désigner une image radiologique dont la nature et la signification restent indéterminées, et dont les principales caractéristiques résident dans les circonstances de sa découverte. Par extension, le terme d'incidentalome a rapidement été utilisé pour désigner toutes sortes d'images permettant la découverte fortuite d'anomalies, qu'il s'agisse d'un anévrisme

née, pourtant présentes sur $5 \%$ des scanners abdominaux $[5,6]$, ce nouveau terme médical, était porteur d'une double indétermination : indétermination de la nature de la masse visualisée et indétermination de sa signification clinique, et, de ce fait, indétermination sur la conduite à tenir au cas par cas. Construit par l'adjonction du suffixe grec « oma » (qui signifie tumeur) derrière l'adjectif anglais « incidental » (qui signifie « qui a un caractère accessoire, secondaire ») [7], le néologisme anglais « incidentaloma »- et sa formulation française « incidentalome »-a donc une double connotation, à la fois concrète et inquiétante, par son suffixe qui renvoie à une tumeur, et conjoncturelle et rassurante, par sa racine qui précise son caractère fortuit et secondaire. Par la suite, les contextes d'usage de ce néologisme ont été étendus. D'abord restreint au domaine de l'endocrinologie, en référence à des images visualisées sur la thyroïde ou I'hypophyse $[8,9]$, le terme « incidentaloma » est ensuite volontiers utilisé pour désigner des masses découvertes fortuitement par l'imagerie (scannographique ou autre) sur d'autres organes aux fonctions aussi variées que le foie, le cœur, les annexes utérines, l'intestin grêle, etc. [10-13]. Dans tous ces usages, la notion véhiculée est celle d'une tumeur de découverte inattendue par l'imagerie et de signification indéterminée au stade de sa visualisation. La persistance de cette situation d'indétermination au fil du temps, et ses conséquences en matière de prise en charge concrète des patients, a conduit la communauté des radiologues à une réflexion scientifique collégiale ayant abouti à l'élaboration de recommandations d'experts à partir de 2010 [14-17]. Il s'agissait alors de donner un sens clinique à un signe radiologique qui continuait de poser des problèmes pratiques aux praticiens près de 20 ans après son identification.

\section{Définir l’incidentalome}

L'élaboration des recommandations de prise en charge a été l'occasion pour les experts de l'American College of Radiology (ACR) de proposer de la veine porte ou encore d'une anomalie du fond de I'œil diagnostiquée par rétinographie $[19,20]$. Au fil du temps, les usages du néologisme lui ont conféré un sens plus abstrait, le dégageant de toute forme concrète. La notion de tumeur, véhiculée par le suffixe «ome », disparaît ou n'est plus obligatoire. Le terme incidentalome semble donc pouvoir désigner toute découverte fortuite et de signification indéterminée, objectivée par un examen de santé prescrit pour un autre objet, et qui va être à l'origine d'un questionnement destiné à lui donner une signification clinique. II est désormais possible de parler d'incidentalome génétique [21] ou d'incidentalome biologique (définis comme «la découverte fortuite d'une anomalie biologique lors d'un examen sanguin prescrit de manière systématique ou pour une autre raison ») [22, 23].

Les usages du terme entrent ainsi en contradiction avec les définitions initialement édictées par les sociétés savantes. Dans la littérature médicale, l'incidentalome ne possède, de fait, aucune définition précise et consensuelle. Pourtant, il semble toujours correspondre aux besoins de médecins qui choisissent arbitrairement de l'utiliser dans leurs propres domaines d'expertise.

\section{La fonction heuristique du néologisme}

Mirilas et Skandalakis ont proposé, en 2002, de bannir le terme incidentalome pour se contenter de décrire simplement les lésions constatées posant question en associant

\footnotetext{
2 Une masse ou une lésion découverte accidentellement, détectée par tomodensitométrie, IRM [imagerie par résonance magnétique] ou autre modalité d'imagerie réalisée pour une raison indépendante.
} 


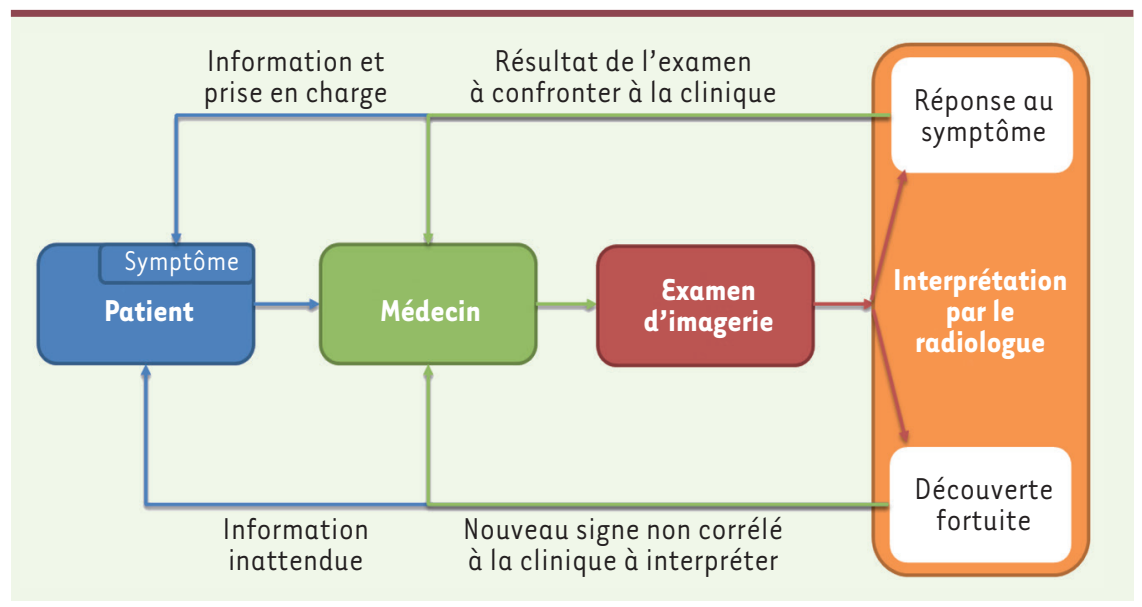

Figure 2. Modifications apportées par les découvertes fortuites (adaptation d'une iconographie avec l'autorisation de M.F. Mamzer).

« de découverte fortuite » à la description [4]. Mais cette proposition est réductrice : elle dénie la portée heuristique du néologisme.

II apparaît aujourd'hui clairement que derrière ce terme se cache bien plus que le côté « incident » d'une découverte. La création de ce néologisme traduit l'émergence d'une nouvelle démarche médicale, portée par la puissance des nouvelles technologies qui modifient la nature des données auxquelles les médecins ont accès dans l'exercice de la clinique. La question sous-jacente est celle de la pertinence de ces données en tant que signe porteur d'une signification clinique qu'il convient de découvrir ou de préciser. II est alors possible d'assister à un retournement complet de la démarche clinique. L'imagerie n'est plus un simple moyen diagnostique permettant l'aboutissement d'une démarche clinique, mais elle est devenue un moyen de générer de nouvelles données, non reliées à un raisonnement clinique antérieur ou tout simplement à une plainte du patient.

La portée heuristique du terme «incidentalome » prend alors tout son sens. L'heuristique est en même temps ce qui sert à la découverte et une méthode pédagogique qui consiste à faire découvrir par l'élève ce que l'on veut lui enseigner. La découverte fortuite en imagerie, à travers le terme «incidentalome», fait découvrir à la médecine une nouvelle forme de clinique. Celle-ci n'est plus fondée sur la plainte du patient mais sur la génération aléatoire de nouvelles données par les technologies médicales de pointe. Ce terme invite donc au questionnement de la médecine sur sa propre capacité à générer des données dont il n'est pas possible, lors de leur découverte, de savoir si elles sont signifiantes ou non, et, surtout, si elles seront lourdes de conséquences pour le patient.

\section{Une clinique des données?}

L'apparition et l'évolution du terme « incidentaloma » est la conséquence du développement d'un nouveau questionnement médical. En effet, le schéma classique de la clinique où le symptôme donnait lieu à la plainte du patient, la plainte à l'examen clinique, l'examen clinique aux éventuels examens spécialisés, les examens au diagnostic et le diagnostic au traitement, est complètement bouleversé. Désormais, la plainte, le symptôme et l'examen clinique du patient peuvent être remplacés par un (ou plusieurs) examen qui permet de générer des données puis, à terme, de poser un diagnostic. Le traitement d'une pathologie peut ainsi débuter avant même qu'un patient ne se «sente » malade, modifiant l'approche de la maladie par la société. La maladie devient alors constitutive de l'être humain, car objectivée mais en dehors de tout ressenti (Figure 2). Comme le vantait si bien le Docteur Knock de Jules Romains: «Les gens bien portant sont des malades qui s'ignorent $\gg[24]$.

Une découverte fortuite en imagerie médicale - mais aussi en génomique - résulte en l'apparition d'une nouvelle donnée qui n'est pas reliée à la plainte du patient qui interroge le médecin en lui demandant un diagnostic en dehors de tout autre élément clinique. Cette donnée est tirée du «silence des organes »-selon l'expression de René Leriche ${ }^{3}$. Ce silence quitte l'intimité du patient pour devenir une donnée de santé par nature extérieure au patient car traitée numériquement (Figure 3). II existe alors une rupture entre le ressenti du patient et les données objectivantes qui naissent des examens technologiques pratiqués. Ainsi, comme le décrit Didier Sicard ${ }^{4}$ dans son ouvrage «La médecine sans le corps », le corps est virtualisé à travers des images numérisées et des chiffres [25]. L'intime du corps anatomique, ignoré même du patient, est alors représenté sous forme de valeurs quantifiables et objectivables qui sont extérieures à la personne humaine mais aussi, de plus en plus souvent, inaccessibles à sa compréhension même, ce qui nécessite l'usage d'algorithmes pour comprendre un corps toujours plus réduit à ses données. L’homme cède alors sa place à son hologramme construit virtuellement par les technologies médicales [30] $(\rightarrow)$.

Les incidentalomes, nés avec l'imagerie numérisée de la $(\rightarrow)$ Voir le Repères de J. Haiech, $m / s$ $n^{\circ} 10$, octobre 2020 , page 919 tomodensitométrie, sont les signes d'une nouvelle forme de médecine qui se propose, sans encore complètement l'assumer, de se passer de l'humain, en se

\footnotetext{
3 « La santé, c'est la vie dans le silence des organes ». Formule de René Leriche (1879-1955), chirurgien et physiologiste français, spécialiste de la douleur.

${ }^{4}$ Professeur de médecine, Didier Sicard a été président du Comité Consultatif
} National d'Éthique de 1999 à 2008. 


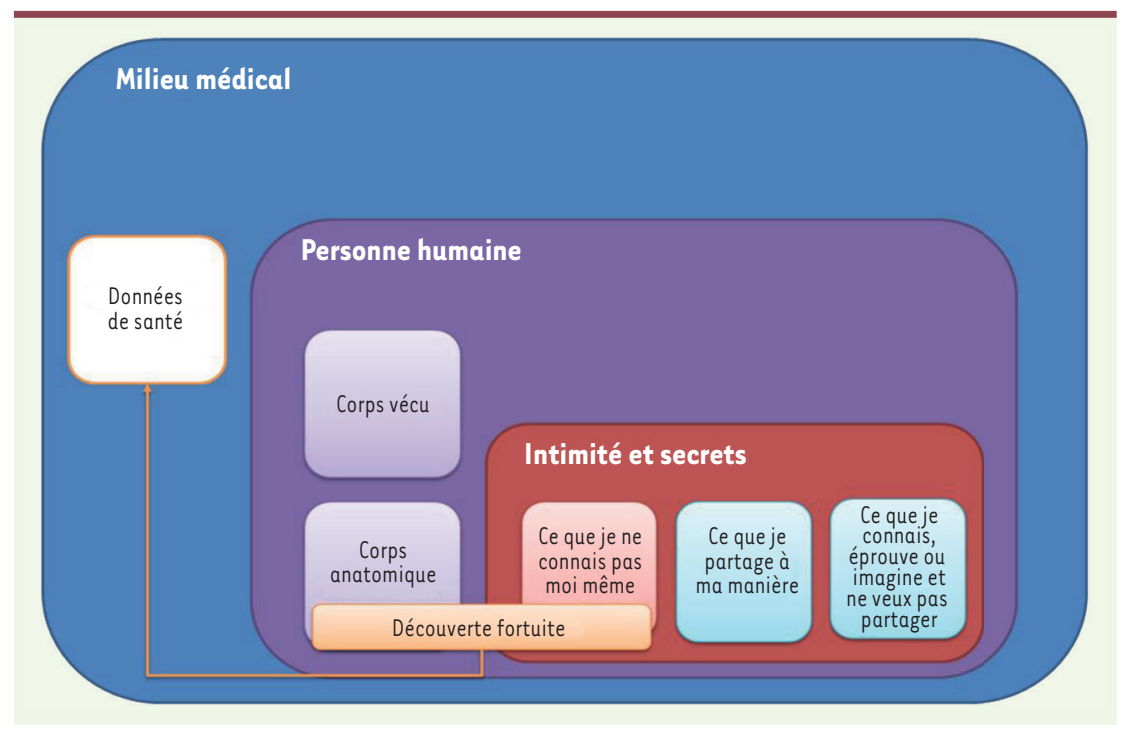

Figure 3. Les découvertes fortuites, de l'intimité aux données de santé (adaptation d'une iconographie avec l'autorisation de M.F. Mamzer).

focalisant uniquement sur les données provenant du corps physique : une clinique des données. Dans cette clinique, les incidentalomes s'expriment alors uniquement comme une nouvelle inconnue présente dans une équation, toujours plus complexe, qui doit être résolue mathématiquement. Ces incidents doivent alors être quantifiés afin de pouvoir corréler la donnée «incidentalome » aux conséquences probables pour le patient.

La recommandation de la Presidential Commission for the Study of Bioethical Issues (PCSBI) portant sur les découvertes fortuites, insiste sur la nécessité de l'anticipation de ces découvertes [26]. Selon cette commission, l'anticipation permettrait une prise en charge optimale de la découverte, et donc la résolution de l'incertitude portée par cette anomalie non corrélée à un symptôme. Depuis près de trente ans, il est néanmoins connu avec certitude que les examens d'imagerie produisent des découvertes fortuites. Pourtant, malgré cette anticipation, les découvertes fortuites ne cessent de faire l'objet de nouvelles publications et de recommandations. L'anticipation générale de la découverte fortuite ne résout donc pas le conflit d'une découverte unique chez un patient tout aussi unique. Il y a sans aucun doute l'intuition des médecins qu'une clinique des données n'est pas une clinique des patients. Ce que soigne un médecin, c'est un homme et non les anomalies quantifiables de son hologramme de données.

\section{Dépasser l'incidentalome}

La découverte fortuite en imagerie est un événement fréquent, quantifiable et désormais anticipé [26]. S’il n'est pas possible de définir, sur un individu, la probabilité qu'un incidentalome se présentera lors d'un examen, aucun radiologue n'ignore, en revanche, le fait que plus la zone explorée est étendue, plus l'âge du patient est élevé, plus seront élevées les probabilités de voir apparaître une image non corrélée à la clinique. Alors, les découvertes fortuites en imagerie médicales le sontelles encore réellement?

En 2013, l'American College of Medical Genetics and Genomics (ACMG) faisait paraître des recommandations provenant d'un groupe de travail interne indiquant comment rapporter les découvertes fortuites provenant d'un séquençage génomique [27]. Cette recommandation pourtant intitulée «ACMG Recommendations for Reporting of Incidental Findings in Clinical Exome and Genome Sequencing » voyait le terme « incidental» associé à « secondary »: « reporting of incidental (or secondary) findings » [27]. Cette double terminologie semble indiquer une hésitation des experts sur la terminologie adéquate. Cette hésitation est cristallisée dans la définition fournie dans la recommandation. Celle-ci décrit, dans un premier temps, la découverte fortuite (ou incidente) comme une «découverte inattendue positive » mais I'ACMG choisit ensuite d'utiliser le terme découverte fortuite comme le « résultat d'une recherche délibérée [...] qui n'est apparemment pas pertinente avec l'indication diagnostique du test » [27]. Cette utilisation du terme découverte fortuite est contradictoire avec l'idée même de fortuité et avec l'usage commun. Trois ans plus tard, lors d'une nouvelle recommandation, L'ACMG a décidé de supprimer le terme « découverte fortuite » pour, finalement, le remplacer par « découverte secondaire » $[28,29]$. Cette terminologie suit la définition de la PCSBI qui décrit la découverte secondaire par : «Le praticien cherche à découvrir $A$, mais recherche aussi activement $D$ par recommandation d'expert » [26].

Ce passage de la découverte fortuite à la découverte secondaire en génétique est propre à interpeller les radiologues. En effet, à l'instar des généticiens réalisant des séquençages complets du génome, ceux-ci scrutent également toutes les données qu'ils produisent lors d'un examen, guidés, mais non limités, par les indications cliniques. Ainsi, ils recherchent activement des découvertes qui ne sont plus fortuites ni même inattendues mais bien secondaires à l'examen.

Cela ne vide, cependant, pas la question de l'agir. Que la découverte soit secondaire ou inattendue, le problème reste similaire: que faire de cette nouvelle information? Quels que soient les domaines, il n'existe pas de réponse unique. Trois exemples représentatifs des efforts des soignants et des institutions ou sociétés savantes en imagerie médicale peuvent être proposés à 
la réflexion : 1) Créer des algorithmes de prise en charge des découvertes [31]. II s'agit alors de suivre un chemin prédéfini suivant les avis d'experts. Cela permet une prise en charge efficace de la découverte mais ne résout pas les problèmes de l'information du patient. 2) Anticiper et communiquer sur la découverte [26]. Cela ne résout pas les problèmes de l'incertitude inhérente à la découverte mais permet de penser une information efficace auprès du patient (que ce soit avant, pendant ou après l'examen). 3) Donner au patient une information modulée par la gravité de la découverte [32]. La proposition est alors, en fonction de la gravité de la découverte, de ne rien dire ou d'envisager des explorations supplémentaires. Cette approche tient compte de l'information du patient mais ne traite pas de l'incertitude que certaines découvertes peuvent susciter.

Aucune de ces approches n'est parfaite. Elles comportent toutes des forces et des faiblesses. Une approche appropriée de l'agir médical en situation de découverte fortuite ou secondaire devra prendre en compte l'information médicale ainsi que son impact sur le patient et proposer une gestion de l'incertitude sur le diagnostic et le pronostic des découvertes réalisées. $\diamond$

\section{SUMMARY}

\section{From incidentaloma to secondary discovery}

The term incidentaloma, created in 1982, has spread throughout medical literature. However, there does not seem to be a precise definition to describe what an incidentaloma is. In the initial uses, the word incidentaloma systematically designated a mass that was detected during an imaging examination prescribed for diagnostic purposes unrelated to the reason for carrying out the examination. Naming this mass "incidentaloma" did not give any precision on its nature as it can be located in many anatomical zones, secreting or not, benign or malignant, etc. Today, the term "incidentaloma" carries a much broader dimension which seems to cover the notion of incidental discovery, whether radiological, biological or genetic. The evolution of the term "incidentaloma" demonstrates its heuristic nature. It is a sign of a change in modern medicine which hesitates between a patient clinic and a data clinic. Fortuitous discoveries are a phenomenon known and anticipated by radiologists. Thus, these discoveries are no longer fortuitous, or even unexpected, but secondary to the use of health technology. $\diamond$

\section{LIENS D'INTÉRÊT}

Les auteurs déclarent n'avoir aucun lien d'intérêt concernant les données publiées dans cet article.

\section{RÉFÉRENCES}

1. Seddon JM, Baranetsky N, Van Boxel PJ. Adrenal incidentalomas: need for surgery. Urology $1985 ; 25: 1-7$.

2. Prinz RA, Brooks MH, Churchill R, et al. Incidental asymptomatic adrenal masses detected by computed tomographic scanning: is operation required? JAMA $1982 ; 248: 701-4$.

3. Geelhoed GW, Druy EM. Management of the adrenal incidentaloma. Surgery $1982 ; 92: 866-74$.

4. Mirilas P, Skandalakis JE. Benign anatomical mistakes: incidentaloma. Am Surg $2002 ; 68$ : 1026-8.

5. Blake MA, Cronin CG, Boland GW. Adrenal imaging. AJR Am J Roentgenol 2010 ; 194 : 1450-60.

6. Song JH, Chaudhry FS, Mayo-Smith WW. The incidental adrenal mass on CT: prevalence of adrenal disease in 1,049 consecutive adrenal masses in patients with no known malignancy. AJR Am J Roentgenol 2008 ; $190: 1163-8$.
7. https://www.le-tresor-de-la-langue.fr/definition/incident-l

8. Molitch ME, Russell $\varepsilon$ J. The pituitary incidentaloma. Ann Intern Med 1990 ; $112: 925-31$.

9. Armasu I, Ciobanu D, Preda C, et al. L'incidentalome thyroïdien - problèmes palpables des nodules impalpables. Ann Endocrinol $2014 ; 75: 512-3$.

10. Little JM, Kenny J, Hollands MJ. Hepatic incidentaloma: a modern problem. World J Surg $1990 ; 14: 448-51$.

11. Collu $\varepsilon$, Grimaldi A, Benussi S, et al. A rare case of unexpected cardiac incidentaloma causing syncope. HSR Proc Intensive Care Cardiovasc Anesth $2010 ; 2: 225-7$.

12. Spencer JA, Gore RM. The adnexal incidentaloma: a practical approach to management. Cancer Imaging $2011 ; 11: 48$.

13. Donnellan F, Moran S, Patchett SE. An incidentaloma at ileal intubation. World J Gastrointest Endosc 2011 ; 3 : 228-30.

14. Berland LL, Silverman SG, Gore RM, et al. Managing incidental findings on abdominal CT: white paper of the ACR incidental findings committee. J Am Coll Radiol $2010 ; 7: 754-73$.

15. Hoang JK, Langer JE, Middleton WD, et al. Managing incidental thyroid nodules detected on imaging: white paper of the ACR incidental thyroid findings committee. J Am Coll Radiol 2015 ; 12 : 143-50.

16. https://www.acr.org/-/media/ACR/Files/Practice-Parameters/ CommunicationDiag.pdf

17. https://www.rcr.ac.uk/publication/management-incidental-findingsdetected-during-research-imaging

18. Berland LL, Silverman SG, Gore RM, et al. Managing incidental findings on abdominal CT: white paper of the ACR incidental findings committee.J Am Coll Radiol $2010 ; 7: 754-73$.

19. Guilbaud T, Birnbaum DJ, Duconseil P, et al. Portal vein aneurysm incidentaloma. Surgery $2017 ; 162: 1177-8$.

20. Bettembourg 0 , Benchekroun S, Le Jeune M, et al. Évaluation du dépistage de la rétinopathie diabétique par rétinophotos et télétransmission. J Fr Ophtalmol 2005; $28: 180$.

21. Kohane IS, Masys DR, Altman RB. The incidentalome: a threat to genomic medicine. JAMA $2006 ; 296: 212-5$.

22. Le Mouel $\varepsilon$. Incidentalomes biologiques : étude prospective en consultation de médecine interne. Rev Med Interne 2011 ; 32 : S264.

23. Lienhardt-Roussie A, Simonin G. Anomalies des phosphatases alcalines. https://pap-pediatrie.fr/biologie-genetique/anomalies-desphosphatases-alcalines

24. Romains J. Knock ou le triomphe de la médecine. Paris : Gallimard. 1924. 25. Sicard D. La médecine sans le corps. Paris: Plon. 2002 : 280 p.

26. Presidential commission for the study of bioethical issues. Anticipate and communicate, ethical management of incidental and secondary findings in the clinical, research, and direct-to-consumer contexts. Washington DC : Bioethics commission, 2013.

27. Green R, Berg J, Grody W, et al. ACMG recommendations for reporting of incidental findings in clinical exome and genome sequencing. American College of Medical Genetics and Genomics, 2013.

28. Kalia SS, Adelman K, Bale SJ, et al. Recommendations for reporting of secondary findings in clinical exome and genome sequencing, 2016 update (ACMG SF v2.0): a policy statement of the American college of medical genetics and genomics. Genet Med 2017 ; $19: 249-55$.

29. Green RC, Berg JS, Grody WW, et al. ACMG Recommendations for reporting of incidental findings in clinical exome and genome sequencing. Genet Med $2013 ; 15: 565-74$.

30. Haiech J. Parcourir I'histoire de l'intelligence artificielle, pour mieux la définir et la comprendre. Med Sci (Paris) $2020 ; 36: 919-23$.

31. loachimescu A, Remer $\varepsilon$, Hamrahian A. adrenal incidentalomas: a disease of modern technology offering opportunities for improved patient care. Endocrinol Metab Clin North Am 2015 ; 44 : 335-54.

32. Claeys A, Vialatte JS. Rapport sur l'impact et les enjeux des nouvelles technologies d'exploration et de thérapie du cerveau. Office parlementaire d'évaluation des choix scientifiques et technologiques. Sénat-Assemblée Nationale, 2012 : Rapport $n^{\circ}$ 476. https://www.senat. $\mathrm{fr} /$ notice-rapport/2011/rl1-476-1-notice.html

TIRÉS À PART

C. Gesbert 